\title{
Structure Is Informative: On Mining Structured Information Networks
}

\author{
Jiawei Han \\ Department of Computer Science, \\ University of Illinois at Urbana-Champaign \\ hanj@cs.uiuc.edu
}

Many objects in the real world are interconnected, forming complex information networks. There have been a lot of studies on mining homogeneous information networks where objects and links are either treated as of the same type, such as friends linking with friends, or treated indiscriminatively, without structural or type distinction. However, real-world objects and links often belong to distinct types, such as students, professors, courses, departments, teach and advise in a university network, and such typed networks form structured, heterogeneous information networks.

We explore methodologies on mining such structured information networks and introduce several interesting new mining methodologies, including integrated ranking and clustering, classification, role discovery, data integration, data validation, and similarity search. We show that structured information networks are informative, and link analysis on such networks becomes powerful at uncovering critical knowledge hidden in large networks. 